or large communities, 'slow burners' whose impact grows gradually or suddenly after a delay, and so on.

Such isolated statistics serve to illustrate a point that has been more systematically documented in the bibliometrics literature. Take, for example, an analysis of the correlation between judgements of scientific value using metrics, including citations, and those using peer review, in condensed-matter physics (E. J. Rinia et al. Res. Policy 27, 95-107; 1998). The study found disagreements in judgement between the two methods of evaluation in $25 \%$ of the 5,000 papers examined. In roughly half of these cases, the experts found a paper to be of interest when the metrics did not, and in the other half, the opposite was the case. The reasons for the differences are not fully understood.

It is also important to note that the use of metrics as an evaluation method does not have widespread support within the scientific community. Some members of the expert panels judging work in this year's RAE warn of the dangers to the quality of research assessment under a metrics-based model. These fears were expressed even by experts in subject areas thought to be most appropriate for metricsbased assessment, such as biology and chemistry. Metrics are not well established for the applications of science, or for disciplines less dependent on journal publication.

Britain is not alone in encountering problems in developing robust metric indicators of research quality. The Australian government is also dealing with a backlash from some universities and leading researchers against its current attempts to do the same thing.

The signs are that, after several false starts and delays, the final proposals for the REF, due in autumn 2009 , are unlikely to be the radical departure from the RAE that the government first envisaged in 2006. Expert review is far from a problem-free method of assessment, but policy-makers have no option but to recognize its indispensable and central role.

\title{
A public service
}

\section{The Christmas bird count is a model to be emulated in distributed, volunteer science.}

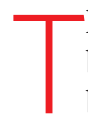
he 5th of January marks the completion of the 109th Christmas bird count, a yearly rite in which groups of North American bird-lovers pick a day around the winter solstice, fan out in teams to their designated areas, and count every bird that they see.

Held every year since 1900, when the National Audubon Society proposed it as an alternative to the then-popular Yuletide activity of competitively shooting birds, the count is the longest-running volunteer science project in the world. Its data have informed reams of peer-reviewed work, such as an ongoing effort by Audubon researchers to predict how birds will adjust their ranges in response to climate change.

The count has served as a model for any number of volunteer science efforts. Such projects now flourish - not least because the Internet makes it so easy for scientists to find, recruit and coordinate the volunteers. Out in the field, examples range from Project BudBurst, in which participants report on the timing of climate-influenced botanical events such as flowering and leafing, to the Great World Wide Star Count, in which astronomy buffs check the number of stars visible in certain bright constellations as a way of monitoring light pollution.

Indoors, meanwhile, network-based projects include Folding@home, in which millions of users allow their idle home computers to be used to simulate protein folding, and Galaxy Zoo, in which participants use their prowess at pattern recognition to classify the millions of galaxies captured in telescopic images - something that still flummoxes computers.

The lesson of this list is that the world is full of enthusiastic people and that the opportunities for researchers to tap into this enthusiasm are limited only by their own imaginations. Volunteer science is a win-win situation for all concerned. Scientists get to take on projects that would not be feasible for even the largest research group, while helping to increase the public's understanding of, and support for, science. And the volunteers get to have fun, while experiencing the satisfaction of defending the environment, fighting disease or expanding human knowledge.

So researchers should think creatively about whether the data they need, or the crunching or sorting they must do, can be outsourced to members of the public. And while they are at it, perhaps they should also consider joining one or more citizen science projects themselves. Participation in such efforts can reconnect scientists consumed with grant-writing and project management with the 'doing' of science. In the Christmas bird count, the most skilled bird spotters and identifiers are inevitably the non-scientists; professional ornithologists spend too much time doing paperwork. And, of course, volunteering for science feels good, especially when you see a black oystercatcher, say, or two merging galaxies - something fun, beautiful and rare.

\section{ANNOUNCEMENT \\ Evolutionary gems}

About a year ago, an Editorial in these pages urged scientists and their institutions to 'spread the word' and highlight reasons why scientists can treat evolution by natural selection as, in effect, an established fact (see Nature 451, 108; 2008).

This week we are following our own prescription. Readers will find at www.nature.com/evolutiongems a freely accessible resource for biologists and others who wish to explain to students, friends or loved ones just what is the evidence for evolution by natural selection. Entitled ' 15 evolutionary gems', the document summarizes 15 lines of evidence from papers published in Nature over the past 10 years. The evidence is drawn from the fossil record, from studies of natural and artificial habitats, and from research on molecular biological processes.

In a year in which Darwin is being celebrated amid uncertainty and hostility about his ideas among citizens, being aware of the cumulatively incontrovertible evidence for those ideas is all the more important. We trust that this document will help. 